\title{
Canadian guideline on HIV pre-exposure prophylaxis and nonoccupational postexposure prophylaxis
}

\author{
Darrell H. S. Tan MD PhD, Mark W. Hull MD MHSc, Deborah Yoong BScPhm PharmD, Cécile Tremblay MD, \\ Patrick O’Byrne RN (EC) PhD, Réjean Thomas MD, Julie Kille RN, Jean-Guy Baril MD, Joseph Cox MD MSc, \\ Pierre Giguere BPharm MSc, Marianne Harris MD, Christine Hughes BScPharm PharmD, Paul MacPherson MD PhD, \\ Shannon O’Donnell MD MPH, Joss Reimer MD MPH, Ameeta Singh BMBS MSc, Lisa Barrett MD PhD, \\ Isaac Bogoch MD MSc, Jody Jollimore MPP, Gilles Lambert MD, Bertrand Lebouche MD PhD, Gila Metz MD, \\ Tim Rogers PhD, Stephen Shafran MD; for the Biomedical HIV Prevention Working Group of the CIHR Canadian \\ HIV Trials Network
}

Cite as: CMAJ 2017 November 27;189:E1448-58. doi: 10.1503/cmaj.170494

CMAJ Podcasts: author interview at https://soundcloud.com/cmajpodcasts/170494-guide

$\mathbf{N}$ ew HIV infections occur every year in Canada, ${ }^{1}$ highlighting the need for integrated prevention programs. Pre-exposure prophylaxis (PrEP) and nonoccupational postexposure prophylaxis (nPEP) are two important strategies for preventing HIV that should be considered standard of care and implemented as components of a comprehensive response to the epidemic. Pre-exposure prophylaxis is the use of certain antiretroviral medications by HIV-uninfected persons who are at high, ongoing risk of HIV acquisition, beginning before and continuing after potential HIV exposures. Postexposure prophylaxis (PEP) involves 28 days of antiretroviral medications immediately after a specific HIV exposure, and is "nonoccupational" (nPEP) when used after sexual and injection drug use exposures, rather than accidental exposures that occur in work contexts (e.g., health care).

The risk of HIV acquisition from an exposure depends on the likelihood the source has transmissible HIV infection (Table 1), ${ }^{2-4}$ which we categorize as substantial, low but nonzero, and negligible or none, and the biological risk of HIV transmission based on the exposure type, which we categorize as high, medium or low (Table 2). ${ }^{5}$ We distinguish between three categories for the likelihood that a person has transmissible HIV infection: substantial, low but nonzero, and negligible or none. The categories for the likelihood that a source has transmissible HIV infection depend on the person's HIV treatment status if known to be HIV positive, or on the probability of the person being HIV positive if HIV status is unknown.

The full guideline is available in Appendix 1 (at www.cmaj.ca/ lookup/suppl/doi:10.1503/cmaj.170494/-/DC1).

\section{Scope}

This guideline is applicable to adults who are at risk for acquiring HIV infection through sexual activity or injection drug use, but

\section{KEY POINTS}

- Pre-exposure prophylaxis (PrEP) involving daily tenofovir disoproxil fumarate/emtricitabine $300 / 200 \mathrm{mg}$, taken orally, is a highly effective strategy for reducing the risk of HIV acquisition in adults who are at high, ongoing risk of infection.

- In gay, bisexual and other men who have sex with men with frequent exposures, an on-demand regimen may also be considered.

- Nonoccupational postexposure prophylaxis (nPEP) involving 28 days of antiretroviral medications is an effective strategy for reducing the risk of HIV acquisition from a recent (within $72 \mathrm{~h}$ ) incident of moderate or high-risk exposure to HIV.

- PrEP and nPEP should be part of a combination prevention strategy that includes behavioural interventions, such as condoms and counselling on risk reduction.

may be of particular importance in populations where HIV incidence in Canada remains disproportionately concentrated. More than half of new infections (54.3\%) occur in gay, bisexual and other men who have sex with men (MSM), in whom HIV risk is estimated to be 131 times higher than other men. ${ }^{6}$ HIV incidence among people who inject drugs (PWID), people from HIVendemic countries, and Indigenous people is estimated to be 59, 6.4 and 2.7 times higher than in other Canadians, respectively. ${ }^{6}$ National data on HIV incidence among sex workers and their clients are scarce, perhaps in part because sex work is criminalized in Canada; as such, this guideline should be applied to these individuals based on the presence of other risk factors.

We adopted a client perspective, as our primary intended audience is clinicians working in primary care, infectious diseases, emergency medicine, nursing, pharmacy and related disciplines. 
In addition, policy-makers, community organizations and other stakeholders may find this guideline useful for informing policy and programming.

\section{Methods}

The guideline was developed by the Biomedical HIV Prevention Working Group of the CIHR Canadian HIV Trials Network, with funding from the Canadian Institutes of Health Research and inkind support from the CIHR Canadian HIV Trials Network. We followed the GRADE (Grading of Recommendation, Assessment, Development and Evaluation) system, a rigorous and widely accepted methodology for the development of clinical practice guidelines (Box 1).

We first assembled a panel of 25 experts from across Canada who represent diverse disciplines (infectious diseases, primary care, emergency medicine, public health, pharmacy, nursing, community), with invitations from the co-chairs (DHST and MWH)

Table 1: Categories of risk that a person has transmissible HIV infection ${ }^{2-4}$

\begin{tabular}{|l|l|}
\hline Risk & \multicolumn{1}{c}{ Examples } \\
\hline Substantial & HIV positive and viremic (i.e., viral load > \\
& 40 copies/mL) \\
- & HIV status unknown, but from a population \\
& with high HIV prevalence compared with the \\
& general population (e.g., men who have sex \\
& with men, people who inject drugs) \\
& - HIV positive and believed to have a viral load \\
& $<40$ copies/mL; with concomitant sexually \\
& transmitted infection present at the time of \\
& exposure. \\
\hline Low but nonzero & Confirmed HIV negative \\
& HIV positive with confirmed viral load \\
& $<40$ copies/mL and no known sexually \\
& transmitted infections present at time of \\
& exposure \\
Negligible or none & HIV status unknown, general population \\
&
\end{tabular}

Table 2: Risk of HIV transmission per act by exposure type from an HIV-positive source ${ }^{5}$

\begin{tabular}{|llr|}
\hline Level & \multicolumn{1}{c}{ Exposure type } & $\begin{array}{c}\text { Estimated risk } \\
\text { per act, \% }\end{array}$ \\
\hline High & Anal (receptive) & $1.38(1.02-1.86)$ \\
\hline Moderate & Needle sharing & $0.63(0.41-0.92)$ \\
\hline \multirow{2}{*}{ Low } & Anal (insertive) & $0.11(0.04-0.28)$ \\
& Vaginal (receptive) & $0.08(0.06-0.11)$ \\
& Vaginal (insertive) & $0.04(0.01-0.14)$ \\
& Oral sex (giving) & Precise estimates not \\
& Oral sex (receiving) & \\
\hline & Oral-anal contact & \\
\hline & Sharing sex toys & \\
\hline & Blood on compromised skin & \\
\hline
\end{tabular}

on the basis of expertise in HIV prevention; the rationale for selecting each member was circulated within the panel.

The panel was subdivided into five working groups, each focusing on one of the following: indications for PrEP, provision of PrEP, indications for $\mathrm{nPEP}$, provision of $\mathrm{nPEP}$ and additional issues that warrant attention during PrEP and nPEP clinical encounters.

Through teleconferences and electronic communications, each working group articulated specific questions to be addressed; these were refined with feedback from the entire panel. Of these questions, we identified four key questions of interest regarding specific clinical indications and specific drug regimens for PrEP and $\mathrm{nPEP}$, respectively, and we specified key outcomes of interest in rank order of importance for each key question (Appendix 2, available at www.cmaj.ca/lookup/suppl/ doi:10.1503/cmaj.170494/-/DC1).

In January 2016, an information specialist conducted structured searches of MEDLINE, Embase and CINAHL to address each question, combining terms for PrEP and nPEP with terms for our study designs of interest (clinical trials and cohort studies); this was updated in November 2016 and September 2017. Each retrieved abstract was reviewed for relevance by at least two panel members, and articles were selected for retrieval by consensus of the two reviewers if they were clinical trials or cohort studies of PrEP or nPEP reporting on our outcomes of interest. Each article was reviewed by at least two panel members for evidence relevant to the guideline questions. Findings were extracted onto standardized electronic forms and discussed in the working groups, with critical appraisal of the quality of the evidence according to the GRADE system. ${ }^{7}$ The study selection diagrams for our key questions are presented in Appendix 3, available at www.cmaj.ca/lookup/suppl/doi:10.1503/ cmaj.170494/-/DC1. Summary of findings tables are presented in

\section{Box 1: GRADE system for recommendations ${ }^{7}$}

This guideline was developed using the GRADE (Grading of Recommendation, Assessment, Development and Evaluation) system (www.gradeworkinggroup.org), which specifies two categories of strength of recommendation and four categories of quality of evidence on which recommendations are based.

\section{Strength of recommendation}

- Strong: A strong recommendation is one for which the panel is confident that the desirable effects of an intervention outweigh undesirable effects (or vice versa), across the range of patients for whom the recommendation is intended.

- Weak: A weak recommendation is an action that should be considered, for which the panel is less confident of the balance between desirable and undesirable consequences. Although the majority of individuals in this situation would want the suggested course of action, many would not, and clinicians must recognize that different choices will be appropriate for different individuals.

\section{Quality of evidence}

- High (starting point for randomized controlled trials)

- Moderate

- Low (starting point for observational studies)

- Very low 
Appendix 4, available at www.cmaj.ca/lookup/ suppl/doi:10.1503/cmaj.170494/-/DC1.

Working groups formulated the preliminary wording and grading for each recommendation, after consideration of the overall certainty of the evidence, desirable and undesirable outcomes, patient values, resource requirements and feasibility. To agree upon the wording and grading, we held an in-person panel meeting in Toronto on Apr. 15-16, 2016, followed by a series of teleconferences and electronic discussions. The final statements were approved through consensus rather than through a formal voting process. Formal endorsements were sought from several national organizations.

\section{Management of competing interests}

All panel members agreed to terms of reference that included disclosure of all perceived and actual competing interests to the entire panel at the beginning and end of the guideline development process. Panellists with competing interests were permitted to participate in panel discussions without restriction.

\section{Recommendations}

A summary of the recommendations is in Box 2. A full discussion of the evidence supporting each recommendation is available in Appendix 1. Box 3 outlines factors that should be part of a health systems approach to PrEP and nPEP.

\section{Pre-exposure prophylaxis}

\section{Indications}

PrEP is recommended for MSM (strong recommendation; high quality of evidence) and transgender women (strong recommendation; moderate quality of evidence) who report condomless anal sex within the last six months and who have any of the following:

- Infectious syphilis or rectal bacterial sexually transmitted infection (STI), particularly if diagnosed in the preceding 12 months;

- Recurrent use of nPEP (more than once);

- Ongoing sexual relationship with HIVpositive partner with substantial risk of transmissible HIV; or

- High-incidence risk index (HIRI)-MSM risk score $\geq 11$ (Appendix 1, supplemental Table 2).

PrEP is not recommended in the context of a stable closed relationship with a single

\section{Box 2: Summary of recommendations}

\section{Pre-exposure prophylaxis (PrEP)} Indications

\section{Men who have sex with men (MSM)}

- PrEP is recommended for MSM (strong recommendation; high quality of evidence) and transgender women (strong recommendation; moderate quality of evidence), who report condomless anal sex within the last six months and who have any of the following:

- Infectious syphilis or rectal bacterial sexually transmitted infection (STI), particularly if diagnosed in the preceding 12 months;

- Recurrent use of nonoccupational postexposure prophylaxis (nPEP) (more than once);

- Ongoing sexual relationship with HIV-positive partner with substantial risk of transmissible HIV; or

- High-incidence risk index (HIRI)-MSM risk score $\geq 11$ (Appendix 1, supplemental Table 2).

- PrEP is not recommended in the context of a stable closed relationship with a single partner with no or negligible risk of having transmissible HIV (strong recommendation; moderate quality of evidence).

\section{Heterosexual exposure}

- We recommend PrEP for the HIV-negative partner in heterosexual serodiscordant relationships reporting condomless vaginal or anal sex where the HIV-positive partner has a substantial risk of having transmissible HIV (strong recommendation; high quality of evidence).

- PrEP may be considered for the HIV-negative partner in heterosexual serodiscordant relationships reporting condomless vaginal or anal sex, where the HIV-positive partner has a low but non-negligible risk of having transmissible HIV (weak recommendation; moderate quality of evidence).

People who inject drugs (PWID) exposure

- PrEP may be considered for PWID if they share injection drug use paraphernalia with a person with a non-negligible risk of HIV infection (weak recommendation; moderate quality of evidence).

\section{Regimens}

- We recommend the following regimen for use as PrEP: tenofovir disoproxil fumarate/ emtricitabine (TDF/FTC) 300/200 mg once daily (strong recommendation; high quality of evidence).

- As an alternative, TDF/FTC 300/200 mg administered "on demand" (two pills taken together 2 to 24 hours before first sexual exposure, followed by one pill daily until 48 hours after last sexual activity) may be considered in MSM (weak recommendation; high quality of evidence).

\section{Nonoccupational postexposure prophylaxis}

\section{Indications}

- We recommend nPEP for HIV-negative individuals who present no later than 72 hours after an exposure that is moderate or high risk for HIV transmission with a person who has a substantial risk of having transmissible HIV (strong recommendation; low quality of evidence).

- nPEP can be considered for HIV-negative individuals who present no later than 72 hours after an exposure that is moderate or high risk for HIV transmission with a person who has a low but non-negligible risk of having transmissible HIV (weak recommendation; low quality of evidence).

- We recommend beginning nPEP as soon as possible after an exposure, up to a maximum of 72 hours afterward (strong recommendation; very low quality of evidence).

\section{Regimens}

- The following are recommended as first-line regimens for nPEP:

- One TDF/FTC tablet daily, taken orally, and raltegravir $400 \mathrm{mg}$ twice daily, taken orally, for 28 days (strong recommendation; high quality of evidence); or

- One TDF/FTC tablet daily, taken orally, and dolutegravir $50 \mathrm{mg}$ daily, taken orally, for 28 days (strong recommendation; low quality of evidence); or

- One TDF/FTC tablet daily, taken orally, and darunavir $800 \mathrm{mg}$ daily + ritonavir $100 \mathrm{mg}$ daily, taken orally, for 28 days (strong recommendation; high quality of evidence).

- When the indication for nPEP is clearly established, the full course of PEP may be dispensed from the outset, rather than providing a starter pack (weak recommendation; highquality evidence). 
partner with no or negligible risk of having transmissible HIV (strong recommendation; moderate quality of evidence).

Levels of transmission risk are defined in Table 1. There is highquality evidence that PrEP is effective at preventing HIV among highrisk MSM (Appendix 4). The evidence for its use in transgender women was downgraded to moderate quality because it is primarily extrapolated from data on MSM. To define which MSM and transgender women are at "high risk," we first considered that condomless anal sex is the key risk behaviour driving the high incidence of HIV infection in MSM and transgender women, except in the setting of a monogamous relationship with a partner who has a negligible risk of having transmissible HIV. ${ }^{3}$

The listed criteria were selected, because well-conducted observational studies show that these specific risk factors are associated with a high incidence of subsequent HIV infection among MSM (Appendix 1). The criteria include scoring highly on the HIRI-MSM, ${ }^{8}$ a rigorously developed assessment tool (Appendix 1, supplemental Table 2) that has been validated in at least one Canadian setting. ${ }^{9}$

These recommendations are strong because PrEP has good acceptability, ${ }^{10}$ excellent safety and high effectiveness in this population; because these criteria are readily identifiable by both patients and providers; and because the high risk of HIV infection associated with these criteria implies high cost-effectiveness. Although PrEP is

Box 3: A health systems approach to pre-exposure prophylaxis (PrEP) and nonoccupational postexposure prophylaxis (nPEP) *5

- PrEP and nPEP should be part of a combination prevention strategy that includes behavioural interventions (e.g., condoms, counselling on risk reduction, partner reduction), biomedical interventions (e.g., treatment of HIV-positive partners, testing and treatment of sexually transmitted infection [STI]) and attention to syndemic conditions that may predispose people to increased risk-taking behaviour (e.g., depression, substance use).

- Health systems should ensure the availability of other harm reduction interventions for people who inject drugs, including programs that distribute sterile equipment for drug use and medication-assisted treatments for substance use disorders.

- Health systems should strive to engage a broad number and range of qualified clinical providers in initiating and providing follow-up for PrEP and nPEP, including family and specialist physicians, nurses, nurse practitioners and pharmacists, where provincial scope of practice allows, or under appropriate delegation of responsibility. Nonprescribing health care and service providers should be encouraged to play roles in PrEP and nPEP delivery, including clinical monitoring, screening and management of STIs, counselling on risk reduction and adherence support.

- Medications for nPEP should be readily available in emergency departments, as well as certain clinics (e.g., STI clinics and those serving at-risk populations) where they are likely to be needed urgently.

- PrEP and nPEP providers should be prepared to provide rapid referrals to HIV care for those who test HIV positive during initial assessment or follow-up for PrEP or nPEP.

- HIV-negative people at risk of HIV acquisition, including those who have condomless vaginal or anal sex and people who inject drugs, should be counselled about and considered for PrEP.

*A full discussion and rationale for these statements are provided in Appendix 1. associated with a small risk of renal and bone toxicities, these changes are generally reversible, ${ }^{11,12}$ and we did not feel that the size of these risks warranted a weak recommendation.

We recommend PREP for the HIV-negative partner in heterosexual serodiscordant relationships reporting condomless vaginal or anal sex where the HIV-positive partner has a substantial risk of having transmissible HIV (strong recommendation; high quality of evidence).

PrEP may be considered for the HIV-negative partner in heterosexual serodiscordant relationships reporting condomless vaginal or anal sex, where the HIV-positive partner has a low but non-negligible risk of having transmissible HIV (weak recommendation; moderate quality of evidence).

High-quality evidence has demonstrated high PrEP efficacy in heterosexuals (Appendix 4). Targeting PrEP for those whose partners have a substantial or non-negligible risk of transmissible HIV is supported by the eligibility criteria for the Partners PrEP trial, ${ }^{13}$ as well as data from the Partners Demonstration Project, in which providing PrEP to HIV-uninfected adults in serodiscordant relationships until six months after their HIV-positive partner began antiretroviral therapy was associated with a $96 \%(95 \% \mathrm{Cl}$ [confidence interval] $81 \%-99 \%$ ) reduction in HIV incidence. ${ }^{14}$

Our recommendations focus on heterosexuals in known serodiscordant relationships, because HIV prevalence in the general Canadian heterosexual population is low. ${ }^{1}$ We did not identify any validated assessment tools for predicting incident infection in heterosexual adults in industrialized-world settings such as Canada.

PrEP may be considered for PWID if they share injection drug use paraphernalia with a person with a non-negligible risk of HIV infection (weak recommendation; moderate quality of evidence).

The Bangkok Tenofovir Study, the only randomized controlled trial of PrEP in PWID, showed that daily oral tenofovir disoproxil fumarate (TDF) without emtricitabine (FTC) conferred a 48.9\% (95\% $\mathrm{Cl} 9.6 \%-72.2 \%$ ) reduction in HIV infection; higher efficacy of $74 \%$ was observed among those with detectable concentrations of tenofovir. ${ }^{15}$ This evidence was downgraded to moderate quality because of two main limitations. First, under Thai law, sterile needles could not be provided to study participants, meaning that the incremental benefit of PrEP when a full package of evidence-based prevention strategies for PWID is also implemented remains unknown. Second, it was not possible to distinguish efficacy of PrEP that was attributable to the prevention of sexual versus parenteral HIV transmission, although sexual risk may also be an indication for PrEP as described above. There are also relatively few data on the feasibility, costeffectiveness and acceptability of PrEP in this population. Thus, the recommendation to use PrEP in PWID is weak.

The ARCH-IDU (assessing the risk of contracting HIV among injection drug users) risk assessment tool may be helpful to clinicians who are considering PWID patients for PrEP, ${ }^{16}$ but has not been as rigorously validated as the HIRI-MSM. ${ }^{8}$ PrEP for prevention of HIV infection related to injection drug use is an off-label use of TDF/FTC in Canada.

\section{Regimens}

We recommend the following regimen for use as PrEP: TDF/FTC 300/200 mg once daily (strong recommendation; high quality of evidence). 
Daily TDF/FTC is the PrEP regimen of choice because it has been the most widely evaluated in high-quality studies. ${ }^{13,14,17-19}$ TDF alone, although efficacious in some trials, ${ }^{13,15}$ is not recommended because of its smaller evidence base, and because it offers no major safety ${ }^{20}$ or cost advantage (given the availability of generic TDF/FTC in Canada) over TDF/FTC. Of note, although TDF/FTC did not prevent HIV in two large trials among women in Africa, these negative results were driven by poor adherence to the study drugs. ${ }^{21,22}$ There are no human data on using tenofovir alafenamide/FTC as PrEP, and neither this regimen nor any other available antiretroviral drug can be recommended as PrEP until results of clinical trials become available.

\section{Box 4: Practical advice for providing HIV pre-exposure prophylaxis (PrEP)}

\section{Initial evaluation and monitoring for PrEP}

\section{HIV testing at baseline and follow-up}

- For all people in whom PrEP is being considered or continued, HIV-negative status should be confirmed shortly before every initial or follow-up prescription is provided. This confirmation should involve a laboratory-based fourth-generation assay (or alternative if this is unavailable; Appendix 1, supplemental Table 4). Confirmation of HIV status should further include evaluation for signs or symptoms suggestive of acute HIV infection (Appendix 1, supplemental Box 1) within the last 12 weeks.

- If acute HIV infection is suspected, additional laboratory evaluation with an HIV RNA nucleic acid amplification test (if available) or repeat fourth-generation assay 7 to 21 days later is suggested, and PrEP should be deferred or suspended until results are received.

\section{Renal monitoring}

- Underlying kidney disease should be ruled out before PrEP is started, using a urinalysis and serum creatinine. The estimated glomerular filtration rate should be $>60 \mathrm{~mL} / \mathrm{min}$ for use of PrEP.

\section{Bone health}

- Routine dual-energy $\mathrm{x}$-ray absorptiometry to assess bone mineral density is not advised unless otherwise indicated according to Osteoporosis Canada guidelines at baseline or during PrEP use.

- PrEP may be considered in people with low bone mass or osteoporosis after the risks and benefits have been discussed with them.

\section{Sexually transmitted infections and viral hepatitis}

- Laboratory screening for sexually transmitted infections is suggested at baseline and at each quarterly follow-up visit, with appropriate therapy for any identified infections.

- Hepatitis A, B and C serologies should be performed at baseline, with vaccination for hepatitis A and B for nonimmune individuals and repeat serologic screening every 12 months for those who remain hepatitis $B$ unvaccinated and hepatitis $C$ uninfected.

\section{Frequency of follow-up}

- We suggest follow-up clinical and laboratory evaluation after 30 days and every three months thereafter (Table 3).

- Each PrEP prescription should be for no more than three months, with no automatic refills.

\section{Pregnancy screening}

- We suggest pregnancy screening in people of child-bearing potential using PrEP every three months.

\section{Counselling}

- PrEP clinical encounters should include assessments and counselling regarding strategies for reducing risk of HIV infection, syndemic conditions, potential drug toxicities and adherence to medication.

\section{Adherence support}

- Interventions to support adherence to medication should be discussed at the time that PrEP is begun, actively monitored at every follow-up patient encounter and tailored to the individual patient.

- Specific interventions may include patient counselling, education, medication reminders, behavioural feedback and reinforcement, peer support, follow-up telephone calls or text messages and minimization of out-of-pocket expenses.

\section{PrEP discontinuation}

- We suggest that PrEP be continued for 2 to 28 days after the last HIV exposure.

- Upon PrEP discontinuation, we advise subsequent follow-up HIV testing using a laboratory-based fourth-generation assay when available, or alternative (Appendix 1, supplemental Table 4), at up to eight weeks afterwards.

\section{Special populations \\ Hepatitis B infection}

- If tenofovir disoproxil fumarate/emtricitabine (TDF/FTC) PrEP is prescribed in a person with chronic hepatitis B infection, appropriate monitoring for hepatitis B virus should be performed in accordance with hepatitis B treatment guidelines, if necessary in consultation with a qualified practitioner with experience in treating the virus.

- When considering PrEP discontinuation, the need for ongoing therapy for hepatitis B virus should be assessed. If PrEP is discontinued and no other therapy for hepatitis B virus is used, monitoring for a flare of the condition is advised.

\section{Pregnancy and breastfeeding}

- TDF/FTC PrEP may be considered during pregnancy and breastfeeding after the benefits and risks have been discussed with the patient. 
As an alternative, TDF/FTC 300/200 mg administered "on demand" (two pills taken together 2 to 24 hours before first sexual exposure, followed by one pill daily until 48 hours after last sexual activity) may be considered in MSM (weak recommendation; high quality of evidence).

"On-demand" PrEP has been studied in one randomized placebocontrolled trial among MSM, Intervention préventive de l'exposition aux risques avec et pour les gays (IPERGAY), and showed $86 \%$ efficacy. ${ }^{23}$ This study used a loading dose (two tablets) of TDF/FTC taken 2 to 24 hours before sex, followed by one tablet daily for 48 hours after the last act of sexual intercourse. If sexual activity resumed within a week, a single dose before sex was recommended. If sexual activity resumed more than a week later, then the loading dose schedule (two tablets) was begun again. Of note, participants in this study used a mean of 15 tablets per month, such that the reported efficacy is consistent with the Pre-exposure Prophylaxis Initiative (iPrEx) open-label extension (iPrEx-OLE) finding of very high efficacy even in those who managed to take daily PrEP only four days per week. ${ }^{19}$

The recommendation is weak because there is uncertainty in the effectiveness of "on-demand" dosing for more sporadic sexual exposures (i.e., less than once weekly) among MSM, and no data to guide recommendations for other populations. In contrast to daily PrEP, on-demand dosing is an off-label use of TDF/FTC in Canada.

\section{Practical advice}

Suggestions on how to monitor individuals using PrEP are provided in Box 4 and explained in detail in Appendix 1. The suggested followup schedule is one month after PrEP initiation, followed by a three- monthly visit schedule (Table 3). It is particularly important to document HIV seronegativity before every initial or follow-up PrEP prescription, using the most sensitive locally available assay (fourthgeneration assay or RNA nucleic acid amplification testing; Appendix 1 , supplementary Table 3 ), because undiagnosed HIV is common in populations where PrEP may be indicated, and because PrEP can lead to HIV-drug resistance if taken by a person who is already HIV seropositive. A complete medical history and physical examination should also be performed at each visit, to look for signs and symptoms of acute HIV infection (Appendix 1, supplementary Box 1).

A direct relationship between adherence and HIV prevention efficacy has been clearly shown. Providers should therefore actively discuss and monitor adherence at every encounter with patients, and tailor interventions to support adherence for the individual patient. Although there are only limited data on specific interventions that improve adherence to PrEP, a systematic review of studies across other prevention fields found that multimodal interventions were most effective. ${ }^{24} \mathrm{~A}$ full discussion of interventions that should be considered is available in Appendix 1 (supplementary Box 2).

\section{Nonoccupational postexposure prophylaxis}

\section{Indications}

We recommend nPEP for HIV-negative individuals who present no later than 72 hours after an exposure that is moderate or high risk for HIV transmission with a person who has a substantial risk of having transmissible HIV (strong recommendation; low quality of evidence).

Table 3: Suggested evaluation at baseline and during pre-exposure prophylaxis

\begin{tabular}{|c|c|c|c|c|}
\hline Assay type & Baseline & $30 \mathrm{~d}$ & Q 3 mo & Q $12 \mathrm{mo}$ \\
\hline \multicolumn{5}{|l|}{ Laboratory evaluation } \\
\hline HIV testing* & $\mathrm{X}$ & $x$ & $x$ & \\
\hline Hepatitis A immunity (hepatitis A total antibody) $†$ & $X$ & & & \\
\hline Hepatitis B screen (surface antigen, surface antibody, core antibody) $† \ddagger$ & $x$ & & & $\mathrm{x} \dagger$ \\
\hline Hepatitis C antibody & $\mathrm{X}$ & & & $\mathrm{X}$ \\
\hline $\begin{array}{l}\text { Screening for gonorrhea and chlamydia§ } \\
\text { (urine nucleic acid amplification test, throat and rectal swabs for culture or nucleic } \\
\text { acid amplification; test anatomic sites depending on type of sexual activity reported) }\end{array}$ & $x$ & & $x$ & \\
\hline Syphilis serology§ & $X$ & & $x$ & \\
\hline Complete blood count & $x$ & & & \\
\hline Creatinine & $x$ & $x$ & $x$ & \\
\hline Urinalysis & $x$ & & & \\
\hline Pregnancy test (as appropriate) & $x$ & & $x$ & \\
\hline \multicolumn{5}{|l|}{ Clinical evaluation } \\
\hline Symptoms of HIV seroconversion & $x$ & $x$ & $x$ & \\
\hline PrEP adherence & & $x$ & $x$ & \\
\hline Indication for PrEP & $x$ & $x$ & $x$ & \\
\hline Use of other HIV and STI prevention strategies & $x$ & $x$ & $x$ & \\
\hline Presence and management of syndemic conditions & $x$ & $x$ & $x$ & \\
\hline \multicolumn{5}{|c|}{$\begin{array}{l}\text { Note: PrEP = pre-exposure prophylaxis, STI = sexually transmitted infection. } \\
\text { ^Preferred HIV test is a 4th-generation antibody/antigen combo assay. Those with signs or symptoms of acute HIV should also undergo HIV RNA or poo } \\
\text { test. } \\
\text { †Hepatitis A and/or B vaccine should be initiated in unvaccinated individuals. Those who remain nonimmune to hepatitis B virus should be rescreened } \\
\text { fIndividuals with chronic active hepatitis B should be managed in consultation with an expert on hepatitis B virus according to Canadian guidelines. } \\
\text { §Individuals who have STIs should be offered standard therapy and follow-up as per local guidelines. }\end{array}$} \\
\hline
\end{tabular}


nPEP can be considered for HIV-negative individuals who present no later than 72 hours after an exposure that is moderate or high risk for HIV transmission with a person who has a low but non-negligible risk of having transmissible HIV (weak recommendation; low quality of evidence).

The risk of acquiring HIV depends on the likelihood that the source has transmissible HIV infection (Table 1), and the biological risk of HIV transmission based on the exposure type (Table 2). Among those who present within the 72-hour window during which intervention is possible, nPEP is recommended if the exposure type was moderate-to-high risk and the source individual has a substantial risk of having transmissible HIV infection (Table 4). Although the quality of the evidence regarding PEP efficacy is low, being based on observational studies only (Appendix 1), ethical constraints preclude the potential for higher-quality data in humans.

nPEP is not recommended for individuals who have had a lowrisk exposure, regardless of source HIV status. We also do not recommend nPEP for those who have had a moderate-to-high risk exposure from a source individual who is known to be HIV positive

Table 4: Risk assessment for beginning nPEP initiation*

\begin{tabular}{lll}
$\begin{array}{l}\text { Likelihood that } \\
\text { source person has } \\
\text { transmissible HIV } \\
\text { (from Table 1) }\end{array}$ & High or moderate & \multicolumn{1}{c}{$\begin{array}{c}\text { Risk from exposure type } \\
\text { (from Table 2) }\end{array}$} \\
\hline Substantial & Initiate nPEP & nPEP not required \\
\hline Low & Consider nPEP & nPEP not required \\
\hline Negligible or none & nPEP not required & nPEP not required \\
\hline $\begin{array}{l}\text { Note: nPEP = nonoccupational postexposure prophylaxis. } \\
\text { *Combining risk arising from exposure type and probability that the source has } \\
\text { transmissible HIV to determine when to initiate nPEP. }\end{array}$
\end{tabular}

but is documented to be virologically suppressed on antiretroviral therapy, and who does not have a known concomitant STI. Of note, all PEP use is off label in Canada.

We recommend beginning nPEP as soon as possible after an exposure, up to a maximum of 72 hours afterward (strong recommendation; very low quality of evidence).

Although there are no data on adult humans regarding the maximum time threshold after which nPEP no longer offers protective benefit, data from animal models and the perinatal setting suggest a gradient of prevention benefit, with greater efficacy the sooner that PEP is begun, and no benefit if PEP is started after 72 hours. ${ }^{25,26}$ This recommendation is strong despite the very low quality of evidence, because of its sound basis in the biology of HIV transmission, and because feasibility and ethical constraints preclude the potential for higher-quality human studies.

If possible, assessment of relevant sexual or injection drug use partners is warranted, because ascertainment of their HIV status is key to determining whether nPEP is indicated. However, start of nPEP should not be delayed pending this information. Details on how to conduct this assessment are provided in Box 3, and explained in detail in Appendix 1.

\section{Regimens}

The following are recommended as first-line regimens for $n P E P$ :

- One TDF/FTC tablet daily, taken orally, and raltegravir $400 \mathrm{mg}$ twice daily, taken orally, for 28 days (strong recommendation; high quality of evidence); or

- One TDF/FTC tablet daily, taken orally, and dolutegravir $50 \mathrm{mg}$ daily, taken orally, for 28 days (strong recommendation; low quality of evidence); or

Table 5: Nonoccupational postexposure prophylaxis regimens: preferred and alternate agents*

\begin{tabular}{|c|c|}
\hline Drug category† & Preferred \\
\hline $\begin{array}{l}\text { Two nucleoside } \\
\text { reverse } \\
\text { transcriptase } \\
\text { inhibitors }\end{array}$ & $\begin{array}{l}\text { TDF/FTC } 300 / 200 \text { mg PO once daily } \\
\text { (strong recommendation; low quality } \\
\text { of evidence) }\end{array}$ \\
\hline Third drug & $\begin{array}{l}\text { Darunavir } 800 \text { mg PO once daily + } \\
\text { ritonavir } 100 \text { mg PO once daily } \\
\text { (strong recommendation; high quality } \\
\text { of evidence) } \\
\text { or } \\
\text { Dolutegravir } 50 \text { mg PO once daily } \\
\text { (strong recommendation; low quality } \\
\text { of evidence) } \\
\text { or } \\
\text { Raltegravir } 400 \text { mg PO twice daily } \\
\text { (strong recommendation; high quality } \\
\text { of evidence) }\end{array}$ \\
\hline
\end{tabular}

\section{NOT recommended}

Alternate

Zidovudine/lamivudine 300/150 mg PO twice daily (weak recommendation; low quality of evidence)

or

TDF 300 mg PO once daily + lamivudine 300 mg PO once daily (weak recommendation; low quality of evidence)

Atazanavir $300 \mathrm{mg}$ PO once daily + ritonavir $100 \mathrm{mg}$ PO once daily (weak recommendation; low quality of evidence)

or

Darunavir/cobicistat 800/150 mg PO once daily (weak recommendation; very low quality of evidence)

or

Elvitegravir/cobicistat 150/150 mg (coformulated with TDF/FTC 300/200 mg) PO once daily (weak recommendation; low quality of evidence)

or

Lopinavir/ritonavir 800/200 mg PO once daily (weak recommendation; strong quality of evidence)

or

Raltegravir HD 1200 mg PO once daily (weak recommendation; very low quality of evidence)

Abacavir, didanosine, efavirenz, nevirapine, stavudine

Note: $\mathrm{FTC}=$ emtricitabine, $\mathrm{nPEP}=$ nonoccupational postexposure prophylaxis, $\mathrm{PO}=$ per os (orally), $\mathrm{TDF}=$ tenofovir disoproxil fumarate.

${ }^{*}$ A thorough medication history (including prescription drugs, supplements, herbal preparations) should be taken before selecting an nPEP regimen because of the potential for

drug-drug interactions.

†A complete nPEP regimen includes two nucleoside reverse transcriptase inhibitors plus a third drug. 


\section{Box 5: Practical advice for providing nonoccupational HIV postexposure prophylaxis (nPEP)}

\section{Evaluating the source person}

- If the source person is of unknown HIV status, is available and provides consent, HIV testing with a fourth-generation assay should be performed. If the source is suspected clinically of having acute HIV infection (Appendix 1, supplemental Box 1), then additional laboratory evaluation with an HIV RNA nucleic acid amplification test (if available) or repeat fourth-generation assay 7 to 14 days later is advised.

- If the source person is known to be HIV positive, is available and provides consent, a detailed history of antiretroviral therapy and HIV viral load test should be obtained to guide decisions about the need for and type of nPEP to be provided.

- If the source person is of unknown HIV status but at high epidemiologic risk, or is HIV positive and unavailable or does not provide consent for additional viral load testing (or verification of undetectable status), there should be an assumption of substantial risk for transmissible HIV infection.

\section{Initial evaluation and monitoring for nPEP}

\section{Screening for sexual assault}

- Health care providers who undertake initial assessment for nPEP should distinguish between consensual and nonconsensual exposures and should provide or refer to sexual assault services accordingly.

\section{Baseline HIV testing}

- Baseline HIV status should be determined using a laboratory-based fourth-generation assay when available, or alternative (Appendix 1, supplemental Table 4) for all people in whom nPEP is being considered.

- Where available, an HIV point-of-care test can also be included, but should not replace the standard serology test.

\section{Additional laboratory testing}

- Baseline evaluation of individuals beginning nPEP should include laboratory assessment of hepatic and renal function and evaluation for sexually transmitted infection (STI) and hepatitis infection, with appropriate subsequent management (Table 6).

- Ongoing laboratory monitoring of biochemistry and hematology during nPEP is advised only for those with baseline laboratory abnormalities, or in those who develop signs or symptoms of organ dysfunction or medication-related adverse effects during therapy.

\section{Counselling}

- nPEP clinical encounters should include assessments and counselling regarding strategies for reducing risk of HIV infection, syndemic conditions, potential drug toxicities and adherence to medication.

\section{Adherence support}

- Interventions to support adherence to medication should be discussed when nPEP is begun, actively monitored at every follow-up patient encounter and tailored to the individual patient.

- Specific interventions may include patient counselling, education, medication reminders, behavioural feedback and reinforcement, peer support, follow-up telephone calls or text messages and minimization of out-of-pocket expenses.

\section{Follow-up HIV testing}

- Final follow-up HIV serology should be performed using a fourth-generation assay at 12 weeks after exposure (8 weeks after completion of nPEP).

\section{Stopping nPEP early}

- nPEP should be stopped early if the source tests HIV negative using a fourth-generation assay. However, continuation of nPEP may be considered despite this result where acute HIV infection of the source is strongly suspected based on clinical history (Appendix 1 , supplemental Box 1 ), and results of additional laboratory testing are pending, as previously described.

- nPEP may be stopped early if the source is HIV positive and is found to have had a viral load below the limit of detection ( $<40 \mathrm{copies} / \mathrm{mL}$ ) for $\geq 6$ months with no evidence of concurrent STI at the time of the exposure.

- If $\geq 72$ consecutive hours of nPEP have been missed, stopping nPEP should be considered.

\section{Special populations}

\section{Suspected acute HIV infection}

- If acute HIV infection of the exposed individual is suspected (supplemental Box 1 in Appendix 1), then additional laboratory evaluation with HIV RNA nucleic acid amplification test (if available) or repeat fourth-generation assay 7 to 21 days later should be performed. nPEP should not be withheld pending the results of these investigations. If the exposed individual is subsequently found to be HIV positive and has started on nPEP, the antiretroviral regimen should be continued and an HIV expert should be consulted as soon as possible.

\section{Pre-exposure prophylaxis (PrEP) users}

- We suggest that individuals who are taking PrEP as prescribed (whether as continuous or on-demand use) do not require nPEP after potential HIV exposures.

- In a person who is not using PrEP as prescribed, beginning nPEP may be considered as per the guideline recommendations.

\section{Hepatitis B infection}

- Patients with chronic hepatitis B virus infection who require nPEP may receive a regimen containing tenofovir disoproxil fumarate/emtricitabine (TDF/FTC), but close clinical and laboratory monitoring for hepatitis flares should be considered upon completion of nPEP.

\section{Pregnancy and breastfeeding}

- Patients who are pregnant and require nPEP should receive one TDF/FTC tablet orally daily together with either raltegravir $400 \mathrm{mg}$ orally twice daily or darunavir $800 \mathrm{mg}$ orally daily + ritonavir $100 \mathrm{mg}$ orally daily.

- Breastfeeding during nPEP use is not advised. 
- One TDF/FTC tablet daily, taken orally, and darunavir $800 \mathrm{mg}$ daily plus ritonavir $100 \mathrm{mg}$ daily, taken orally, for 28 days (strong recommendation; high quality of evidence).

Because PEP is highly effective, clinical trials cannot feasibly establish the superiority of any specific nPEP regimen over another for preventing HIV seroconversion. Our recommendations are therefore based primarily on data on rates of regimen completion and adverse events associated with various nPEP regimens (Appendix 4). The recommendations are strong for all three potential regimens because they each have generally favourable risk-benefit profiles, acceptability, costs and feasibility, although the best choice may vary depending on patient characteristics (Table 5 and Appendix 1, supplementary Table 4).

Raltegravir $400 \mathrm{mg}$ twice daily with one TDF/FTC tablet once daily is well tolerated, associated with reasonable adherence, and has a low propensity for drug-drug interactions, but a disadvantage is its twice-daily dosing schedule. ${ }^{27}$ Darunavir $800 \mathrm{mg}$ plus ritonavir $100 \mathrm{mg}$ with one TDF/FTC tablet, all taken once daily, is associated with few adverse effects and high completion rates, and is preferred if there are concerns about potential drug-resistant virus in the source patient or suspected acute HIV infection in the exposed patient; however, this regimen has substantial potential for drug interactions. ${ }^{28}$ Dolutegravir $50 \mathrm{mg}$ with one TDF/FTC tablet, all taken once daily, has minimal disadvantages, but has a limited evidence base for use in nPEP.

Alternative nPEP regimens may be considered (Table 5) and are detailed further in Appendix 1, supplementary Table 4. All nPEP regimens should be taken for 28 days. Although this duration is based on very low-quality data from macaque models, ${ }^{25}$ feasibility and ethical constraints preclude the potential for higher-quality human studies.

When the indication for PPEP is clearly established, the full course of PEP may be dispensed from the outset, rather than providing a starter pack (weak recommendation; high-quality evidence).
A common practice when dispensing nPEP medications is to provide only a partial supply initially (starter pack), enabling prescribers to reassess the need for nPEP when baseline laboratory results become available, modify therapy in cases of drug intolerance or concerns about drug resistance and, ultimately, limit drug costs and toxicities by preventing unnecessary use. However, a systematic review of randomized trials and observational studies showed that dispensing a full course of nPEP rather than a starter kit at initial presentation is associated with fewer PEP refusals and superior PEP completion rates. ${ }^{29}$ This recommendation is weak because variability in who (patients or the institutions that provide the starter packs) covers the cost of the medication in different contexts may lead to differences in which approach is favoured.

\section{Practical advice}

Suggestions on how to deliver nPEP are provided in Box 5 and Table 6 (details provided in Appendix 1). Although human data on the relationship between adherence and efficacy in the setting of nPEP are lacking, animal models show increasing efficacy with an increasing number of days of nPEP use. ${ }^{25}$ As with PrEP, providers should therefore actively discuss and monitor adherence at encounters with patients who are using nPEP, and tailor interventions to support adherence (Appendix 1, supplementary Box 2) for the individual patient.

\section{Implementation}

Although virtually all HIV-negative people highly value avoiding HIV infection, we acknowledge that individuals may have varying preferences regarding the potential for inconveniences, rare drug toxicities and stigma associated with these interventions. To date, medication costs have also restricted the feasibility and acceptability of these strategies. However, the recent introduction of generic TDF/FTC and the increasing availability of public drug coverage for PrEP in Canada

Table 6: Suggested evaluation at baseline, during and after nonoccupational postexposure prophylaxis

\begin{tabular}{|c|c|c|c|}
\hline Test & Baseline & Week 2 & Week 12 \\
\hline HIV testing* & $\mathrm{X}$ & & $\mathrm{x} \dagger$ \\
\hline Hepatitis A immunity (hepatitis A total antibody) $\ddagger$ & $\mathrm{X}$ & & \\
\hline Hepatitis B screen $\ddagger \S$ (surface antigen, surface antibody, core antibody) & $X$ & & \\
\hline Hepatitis C screen (hepatitis C antibody) & $\mathrm{X}$ & & $\mathrm{X}$ \\
\hline $\begin{array}{l}\text { Screening for gonorrhea and chlamydia (urine nucleic acid amplification test; throat } \\
\text { and rectal swabs for culture or nucleic acid amplification; test anatomic sites depending } \\
\text { on type of sexual activity reported) }\end{array}$ & $\mathrm{X}$ & & $\mathrm{X}$ \\
\hline Syphilis serology & $X$ & & $X$ \\
\hline Complete blood count & $\mathrm{X}$ & & \\
\hline Alanine aminotransferase & $X$ & $X^{\star \star}$ & \\
\hline Serum creatinine & $X$ & $X^{\star \star}$ & \\
\hline Pregnancy testing (if appropriate) & $\mathrm{X}$ & & \\
\hline \multicolumn{4}{|c|}{$\begin{array}{l}\text { *Preferred HIV test is a 4th-generation antibody/antigen combo assay. Those with signs or symptoms of acute HIV should also undergo HIV RNA or pooled nucleic acid amplificatio } \\
\text { test. } \\
\text { †Consider repeating HIV serology at } 6 \text { months after exposure if hepatitis C infection was acquired from the exposure. } \\
\text { †Hepatitis A and/or B vaccine should be started in unvaccinated individuals. } \\
\text { §Individuals with chronic active hepatitis B should be referred for hepatitis B virus care as per local guidelines. } \\
\text { †Individuals diagnosed with concurrent sexually transmitted infection during nonoccupational postexposure prophylaxis should be offered standard therapy and follow-up as per } \\
\text { local guidelines. } \\
\text { *^Suggested if abnormal at baseline or symptomatic. }\end{array}$} \\
\hline
\end{tabular}


may have substantial effects on their uptake. Health economic analyses have suggested the high cost-effectiveness of PrEP when targeted to subpopulations who are at greatest risk of HIV infection (e.g., based on highest number of partners or highest HIV incidence). Systematic reviews suggest that nPEP may be cost-effective in industrialized-world settings, but certainty is greatest for higher-risk scenarios (e.g., receptive anal intercourse). ${ }^{30,31}$ In considering these issues, we have made strong recommendations for PrEP and nPEP in patient groups at highest risk of HIV infection, and recommendations to consider these interventions for those at more moderate risk.

Canadian physicians' awareness of PrEP and nPEP has historically been low, although studies on this topic pre-date Health Canada regulatory approval for PrEP. ${ }^{32,33}$ We are currently developing proposals to monitor awareness of, implementation of and fidelity to these guidelines among key stakeholders and will seek funding for these knowledge translation activities in the coming year.

Given the rapidly changing landscape related to HIV prevention, with clinical trials of novel oral, injectable and topical agents in progress and additional studies of alternative dosing strategies and longterm outcomes underway, updates to the guideline are planned when a new product obtains Health Canada regulatory approval for use as PrEP or nPEP in Canada, or within five years of publication.

\section{Other guidelines}

Our recommendations are broadly consistent with major international and industrialized country guidelines. The World Health Organization recommends PrEP for any risk group with HIV incidence higher than 3\%. ${ }^{34}$ More granular recommendations are made for MSM, PWID and heterosexual populations in guidelines from Europe, the United Kingdom, United States and Australia, based on additional risk factors. ${ }^{35-38}$ For MSM, most recommend PrEP for those with a previous STI, and previous nPEP is also included by the International Antiviral Society-USA guideline. In contrast, no other guideline explicitly recommends using the HIRI-MSM tool for targeting PrEP, but because all guidelines recommend PrEP for MSM with history of condomless anal intercourse, a HIRI-MSM score greater than 11 is consistent with these recommendations. Neither the UK or European guidelines recommend PrEP for PWID; however, the UK guideline explicitly recommends access to harm reduction prevention services. For nPEP, overall clinical indications and requirement for a 28-day course of therapy within 72 hours of exposure are similar across guidelines. For all but the Australian guideline, a standard three-drug regimen is recommended, with minor variations in preferred agents.

\section{Gaps in knowledge}

Data are needed from industrialized-world settings on how best to identify individuals at elevated risk of HIV infection (especially non-MSM), on PrEP-related outcomes for populations other than MSM (particularly during pregnancy or breastfeeding), and on intermittent PrEP dosing schedules. Data on the optimal timing of PrEP discontinuation also comprise an important gap.

For nPEP, key knowledge gaps relate to the use of newer antiretroviral agents, strategies for transitioning individuals who are at high risk of HIV infection onto PrEP, and the optimal timing of fol- low-up HIV testing. Research on implementation is greatly needed to understand how best to deliver these complex biobehavioural interventions to at-risk populations as part of a comprehensive strategy for preventing HIV infection.

\section{Conclusion}

The large financial cost of HIV infection and the young age of those newly diagnosed (most new cases occur in those aged 30 to 39 years) ${ }^{1}$ underscore the economic and social importance of preventing new infections. We hope that this guideline will contribute to reducing HIV incidence in Canada by improving the quality of care, increasing access to care, reducing inappropriate variation in practice and promoting the rigorous evaluation of biomedical prevention strategies nationwide.

\section{References}

1. Summary: estimates of HIV incidence, prevalence and proportion undiagnosed in Canada, 2014. Ottawa: Public Health Agency of Canada; 2015.

2. Gray RH, Wawer MJ, Brookmeyer R, et al. Probability of HIV-1 transmission per coital act in monogamous, heterosexual, HIV-1-discordant couples in Rakai, Uganda. Lancet 2001;357:1149-53.

3. Rodger AJ, Cambiano V, Bruun T, et al. Sexual activity without condoms and risk of HIV transmission in serodifferent couples when the HIV-positive partner is using suppressive antiretroviral therapy. JAMA 2016;316:171-81.

4. Cohen MS, Chen YQ, McCauley M, et al. Prevention of HIV-1 infection with early antiretroviral therapy. N Engl J Med 2011;365:493-505.

5. Patel P, Borkowf CB, Brooks JT, et al. Estimating per-act HIV transmission risk: a systematic review. AIDS 2014;28:1509-19.

6. Yang Q, Ogunnaike-Cooke S, Halverson J, et al. Estimated national HIV incidence rates among key populations in Canada, 2014 [abstract EPH35]. Proceedings from the 25th Annual Canadian Conference on HIV/AIDS Research (CAHR), 2016 May 12-15; Winnipeg.

7. Atkins $D$, Best $D$, Briss PA, et al. Grading quality of evidence and strength of recommendations. BMJ 2004;328:1490.

8. Smith DK, Pals SL, Herbst JH, et al. Development of a clinical screening index predictive of incident HIV infection among men who have sex with men in the United States. J Acquir Immune Defic Syndr 2012;60:421-7.

9. Lachowsky N, Cui Z, Sereda P, et al. HIV incidence rate and predictors among gay and other men who have sex with men (MSM) in Vancouver: additional benefit of administrative health data linkage [abstract EPHP 504]. Proceedings from the 25th Annual Canadian Conference on HIV/AIDS Research (CAHR), 2016 May 12-15; Winnipeg.

10. Peng P, Su S, Fairley CK, et al. A global estimate of the acceptability of pre-exposure prophylaxis for HIV among men who have sex with men: a systematic review and meta-analysis. AIDS Behav 2017 Feb. 7 [Epub ahead of print]. doi: 10.1007/ s10461-017-1675-z.

11. Yacoub R, Nadkarni GN, Weikum D, et al. Elevations in serum creatinine with tenofovir-based HIV pre-exposure prophylaxis: a meta-analysis of randomized placebocontrolled trials. J Acquir Immune Defic Syndr 2016;71:e115-8.

12. Mirembe BG, Kelly CW, Mgodi N, et al. Bone mineral density changes among young, healthy African women receiving oral tenofovir for HIV preexposure prophylaxis. J Acquir Immune Defic Syndr 2016;71:287-94.

13. Baeten JM, Donnell D, Ndase P, et al. Antiretroviral prophylaxis for HIV prevention in heterosexual men and women. N Engl J Med 2012;367:399-410.

14. Baeten JM, Heffron R, Kidoguchi L, et al. Integrated delivery of antiretroviral treatment and pre-exposure prophylaxis to HIV-1-serodiscordant couples: a prospective implementation study in Kenya and Uganda. PLoS Med 2016;13:e1002099.

15. Choopanya K, Martin M, Suntharasamai P, et al. Antiretroviral prophylaxis for HIV infection in injecting drug users in Bangkok, Thailand (the Bangkok Tenofovir Study): a randomised, double-blind, placebo-controlled phase 3 trial. Lancet 2013;381:2083-90.

16. Smith DK, Pan Y, Rose CE, et al. A brief screening tool to assess the risk of contracting HIV infection among active injection drug users. J Addict Med 2015; 9:226-32.

17. Grant RM, Lama JR, Anderson PL, et al. Preexposure chemoprophylaxis for HIV prevention in men who have sex with men. NEngl J Med 2010;363:2587-99.

18. McCormack S, Dunn DT, Desai M, et al. Pre-exposure prophylaxis to prevent the acquisition of HIV-1 infection (PROUD): effectiveness results from the pilot phase of a pragmatic open-label randomised trial. Lancet 2016;387:53-60.

19. Grant RM, Anderson PL, McMahan V, et al. Uptake of pre-exposure prophylaxis, 
sexual practices, and HIV incidence in men and transgender women who have sex with men: a cohort study. Lancet Infect Dis 2014;14:820-9.

20. Baeten JM, Donnell D, Mugo NR, et al. Single-agent tenofovir versus combination emtricitabine plus tenofovir for pre-exposure prophylaxis for HIV-1 acquisition: an update of data from a randomised, double-blind, phase 3 trial. Lancet Infect Dis 2014;14:1055-64.

21. Marrazzo JM, Ramjee G, Richardson BA, et al. Tenofovir-based preexposure prophylaxis for HIV infection among African women. N Engl J Med 2015;372:509-18.

22. Van Damme L, Corneli A, Ahmed K, et al. Preexposure prophylaxis for HIV infection among African women. N Engl J Med 2012;367:411-22.

23. Molina JM, Capitant C, Spire B, et al. On-demand preexposure prophylaxis in men at high risk for HIV-1 infection. N Engl J Med 2015;373:2237-46.

24. Marcus JL, Buisker T, Horvath T, et al. Helping our patients take HIV pre-exposure prophylaxis (PrEP): a systematic review of adherence interventions. HIV Med 2014;15:385-95.

25. Tsai CC, Emau P, Follis KE, et al. Effectiveness of postinoculation (R)-9-(2-phosphonylmethoxypropyl) adenine treatment for prevention of persistent simian immunodeficiency virus SIVmne infection depends critically on timing of initiation and duration of treatment. J Virol 1998;72:4265-73.

26. Wade NA, Birkhead GS, Warren BL, et al. Abbreviated regimens of zidovudine prophylaxis and perinatal transmission of the human immunodeficiency virus. N Engl J Med 1998;339:1409-14.

27. Mayer KH, Mimiaga MJ, Gelman M, et al. Raltegravir, tenofovir DF, and emtricitabine for postexposure prophylaxis to prevent the sexual transmission of HIV: safety, tolerability, and adherence. J Acquir Immune Defic Syndr 2012;59:354-9.

28. Fätkenheuer G, Jessen H, Stoehr A, et al. PEPDar: A randomized prospective noninferiority study of ritonavir-boosted darunavir for HIV post-exposure prophylaxis. HIV Med 2016;17:453-9.
29. Ford N, Venter F, Irvine C, et al. Starter packs versus full prescription of antiretroviral drugs for postexposure prophylaxis: a systematic review. Clin Infect Dis 2015;60(Suppl 3):S182-6.

30. Bryant J, Baxter L, Hird S. Non-occupational postexposure prophylaxis for HIV: a systematic review. Health Technol Assess 2009;13:iii, ix-x, 1-60.

31. Hornberger J, Holodniy M, Robertus K, et al. A systematic review of cost-utility analyses in HIV/AIDS: implications for public policy. Med Decis Making 2007; 27:789-821.

32. Sharma M, Wilton J, Senn H, et al. Preparing for PrEP: perceptions and readiness of Canadian physicians for the implementation of HIV pre-exposure prophylaxis. PLoS One 2014;9:e105283.

33. Karris MY, Beekmann SE, Mehta SR, et al. Are we prepped for preexposure prophylaxis (PrEP)? Provider opinions on the real-world use of PrEP in the United States and Canada. Clin Infect Dis 2014;58:704-12.

34. Guideline on when to start antiretroviral therapy and on pre-exposure prophylaxis for HIV. Geneva: World Health Organization; 2015. Available: www.who.int/hiv/pub/ guidelines/earlyrelease-arv/en/ (accessed 2017 Oct. 19)

35. Brady M, Rodger A, Asboe D, et al. Consultation version of the BHIVA/BASHH guidelines on the use of HIV pre-exposure prophylaxis (PrEP) 2017. London (UK): British HIV Assocation; 2017.

36. Wright E, Grulich A, Roy K, et al. Australasian Society for HIV, Viral Hepatitis and Sexual Health Medicine HIV pre-exposure prophylaxis: clinical guidelines. J Virus Erad 2017;3:168-84.

37. Günthard HF, Saag MS, Benson CA, et al. Antiretroviral drugs for treatment and prevention of HIV infection in adults: 2016 recommendations of the International Antiviral Society-USA Panel. JAMA 2016;316:191-210.

38. Guidelines version 8.2 January 2017. London (UK): European AIDS Clinical Society; 2017.
Competing interests: Darrell Tan reports grants from the Canadian Institutes of Health Research and Ontario HIV Treatment Network, during the conduct of the study, and personal fees from Merck and GSK and grants and personal fees from Gilead and ViiV Healthcare, outside the submitted work. Mark Hull reports receiving honoraria for advisory board representation and speaking engagements regarding HIV and the hepatitis $C$ virus from BMS, Gilead, Merck and ViiV Healthcare, paid to his institution. Deborah Yoong reports personal fees from Merck and Gilead Science, outside the submitted work. Cécile Tremblay reports grants and personal fees from Gilead, Merck, ViiV Healthcare, the Canadian Institutes of Health Research and Fonds de recherche santé Québec, both during the conduct of the study and outside the submitted work. Réjean Thomas reports personal fees from Gilead, outside the submitted work. Jean-Guy Baril reports grants from Gilead, Merck and Glaxo, and personal fees from Gilead, Merck and ViiV Healthcare, outside the submitted work. Joseph Cox reports grants and personal fees from ViiV Healthcare, and grants from Merck and Gilead, outside the submitted work. Pierre Giguere reports grants and personal fees from Gilead Sciences, ViiV Healthcare and Merck outside the submitted work. Marianne Harris reports grants and personal fees from Gilead Sciences Canada and personal fees from Merck Canada and ViiV Healthcare, outside the submitted work. Christine Hughes reports grants and personal fees from ViiV Healthcare, and personal fees from Merck Canada and Gilead Sciences Canada, outside the submitted work. Stephen Shafran reports grants from AbbVie, Gilead and Janssen; grants and personal fees from Merck and BMS; and personal fees from Pfizer, outside the submitted work. No other competing interests were declared.

This article has been peer reviewed.

Affiliations: Division of Infectious Diseases (Tan), St. Michael's Hospital, Toronto, Ont.; BC Centre for Excellence in HIV/AIDS (Hull, Harris), Vancouver, BC; Department of Pharmacy (Yoong), St. Michael's Hospital, Toronto, Ont.; Centre de Recherche du Centre Hospitalier de l'Université de Montréal (Tremblay), Montréal, Que.; School of Nursing (O'Byrne), University of Ottawa, Ottawa, Ont.; Clinique L'Actuel (Thomas), Montréal, Que.; Canadian Association of Nurses in HIV/AIDS Care (Kille), Vancouver, BC; Clinique du Quartier Latin (Baril), Montréal, Que.; Direction régionale de santé publique (Cox), Centre intégré universitaire de santé et de services sociaux du Centre-Sud-de-l'île-de-Montréal, Montréal, Que.; Department of Pharmacy (Giguere), The Ottawa Hospital, Ottawa, Ont.; Faculty of Pharmacy \& Phar- maceutical Sciences (Hughes), University of Alberta, Edmonton, Alta.; Division of Infectious Diseases (MacPherson), The Ottawa Hospital, Ottawa, Ont.; Department of Emergency Medicine (O'Donnell), St. Paul's Hospital, Vancouver, BC; Winnipeg Regional Health Authority (Reimer), Winnipeg, Man.; Division of Infectious Diseases, Department of Medicine (Singh, Shafran), University of Alberta, Edmonton, Alta.; Dalhousie University (Barrett), Halifax, NS; Divisions of Internal Medicine and Infectious Diseases (Bogoch), Toronto General Hospital, Toronto, Ont.; Health Initiative for Men (Jollimore), Vancouver, BC; Institut national de santé publique du Québec (Lambert), Montréal, Que.; Research Institute of the McGill University Health Centre (Lebouche), Montréal, Que.; CATIE (Canadian AIDS Treatment Information Exchange) (Rogers), Toronto, Ont.

Contributors: Darrell Tan and Mark Hull conceived the project; Darrell Tan, Mark Hull, Deborah Yoong, Cécile Tremblay, Patrick O'Byrne and Gilles Lambert secured funding; Réjean Thomas, Cécile Tremblay, Patrick O'Byrne, Deborah Yoong and Julie Kille chaired working groups; all authors jointly crafted the recommendations; and Darrell Tan and Mark Hull wrote the first draft of the synopsis. All of the authors revised the manuscript critically for important intellectual content, gave final approval of the version to be published and agreed to be accountable for all aspects of the work.

Funding statement: The development of this guideline was supported by a grant from the Canadian Institutes of Health Research (funding reference number PCS -142089) and in-kind support from the CIHR Canadian HIV Trials Network.

Acknowledgements: The panel thanks David Lightfoot for his assistance with designing and conducting the electronic literature search, Alex Schnubb for his assistance with coordinating the panel meetings and Kevin Pendergraft for his assistance with coordinating community feedback. Darrell Tan is supported by a New Investigator Award from the Canadian Institutes of Health Research and Ontario HIV Treatment Network.

Endorsements: Association of Medical Microbiology and Infectious Disease Canada; Canadian Association of Emergency Physicians; Canadian Association of Nurses in HIV/AIDS Care; Canadian HIV/AIDS and Viral Hepatitis Pharmacists Network; Canadian Public Health Association.

Correspondence to: Darrell Tan, darrell.tan@gmail.com 